Marco Tulio, R. S., Gianny, B. O., Vanessa del Carmen, P. P., Asdrubal Antonio, A. A., \& Ferley, R. G. (2020). Análisis

de las habilidades de pensamiento crítico en los foros académicos virtuales. Teknos Revista Científica, 2020(2), 69-76.

\title{
Análisis de las habilidades de pensamiento crítico en los foros académicos virtuales
}

\section{Analysis of critical thinking skills in virtual academic forums}

\section{Marco Tulio Rodríguez Sandoval', Gianny Bernal Oviedo', Vanessa del Carmen Pérez Peñaloza', Asdrubal Antonio Atencia Andrade ${ }^{1}$, Ferley Ramos Geliz ${ }^{1}$}

\author{
${ }^{1}$ Docente Investigador, Facultad de Humanidades y Educación Corporación Universitaria del Caribe Sincelejo - Colombia \\ marco.rodriguez@cecar.edu.co, giany.bernal@cecar.edu.co, vanessa.perezpe@cecar.edu.co, \\ asdrubal.atencia@cecar.edu.co, ferley.ramos@cecar.edu.co
}

Recibido: 15/ago/2020 - Revisado: 30/sep/2020

Aceptado: 30/oct/2020 - Publicado: 30/dic/2020

\begin{abstract}
Resumen El presente estudio tiene el propósito de analizar las habilidades de pensamiento crítico, a través de la participación en los foros virtuales del curso Ecología Humana en la plataforma Moodle de CECAR. Se utilizó una metodología cualitativa, con trabajo de campo basado en la lectura de contexto de las instituciones educativas, a partir del análisis del contenido del texto, atendiendo al enfoque fenomenológico hermenéutico, orientando el análisis de la descripción e interpretación de la experiencia que vivieron los estudiantes. El análisis de las habilidades de pensamiento crítico se realizó a través de las dimensiones planteadas por Halpern (2006) razonamiento verbal, análisis de argumentos, comprobación de hipótesis, probabilidad de incertidumbre, toma de decisiones y solución de problemas, a través de una matriz de análisis desde las categorías relacionadas con las prácticas ambientalistas y la articulación de la dimensión ambiental en el currículo escolar. Se analizaron las habilidades de pensamiento crítico, logrando las siguientes consideraciones: los estudiantes en los diferentes análisis de los microrrelatos realizan afirmaciones que están respaldadas por evidencias, lo cual, permite generar conclusiones justificadas para la toma de decisiones y la solución de problemas, evidenciando las habilidades de pensamiento crítico para la construcción de diálogos argumentativos y pragmáticos.
\end{abstract}

Palabras claves foros virtuales; diálogos argumentativos; pensamiento crítico; comunidades letradas; ambientes virtuales.

\begin{abstract}
The purpose of this study is to analyze the critical thinking skills through the construction of argumentative and pragmatic dialogues in the virtual academic forums of the Human Ecology course in the Moodle platform of the Corporación Universitaria del Caribe. A qualitative methodology was used, with field work based on the reading of the context of the educational institutions from the analysis of the content of the text, attending to the hermeneutic phenomenological approach, guiding the analysis of the description and interpretation of the experience lived by the students. The analysis of critical thinking skills was carried out through the dimensions proposed by Halpern (2006) [1] verbal reasoning, argument analysis, hypothesis testing, probability of uncertainty, decision making and problem solving through an analysis matrix from the categories related to environmental practices and the articulation of the environmental dimension in the school curriculum. Critical thinking skills were analyzed through the strategy of academic forums in the Human Ecology course, achieving the following considerations: students in the different analyses of the micro-stories make statements that are supported by evidence shown through indicators, which allows generating conclusions mostly justified for decision making and problem solving, evidencing critical thinking skills in the text that is generated through the argumentative dialogue among the participants.
\end{abstract}

Keywords virtual forums; argumentative dialogues; critical thinking; literate communities; virtual environments.

Teknos Revista Científica. | Volumen 20 No.2 - diciembre 2020 | ISSN 1900-7388 (papel) | ISSN 2539-2190 (digital)

DOI: https://doi.org/10.25044/issn.2539-2190 
Marco Tulio, R. S., Gianny, B. O., Vanessa del Carmen, P. P., Asdrubal Antonio, A. A., \& Ferley, R. G. (2020). Análisis de las habilidades de pensamiento crítico en los foros académicos virtuales. Teknos Revista Científica, 2020(2), 69-76.

\section{Introducción}

El pensamiento crítico está relacionado con la lectura y reflexión del contexto, esto le permite a los estudiantes la creación de discursos pragmáticos para exponer un juicio valorativo desde sus vivencias y experiencias, en cuanto a un tema propio de su área disciplinar; lo expuesto, posibilita la escritura epistémica utilizando la argumentación como un recurso que facilita la toma de decisiones con pruebas fehacientes y razonadas para la construcción de comunidades letradas, en las que se construye conocimiento científico.

El objetivo de este trabajo es analizar en los foros académicos virtuales del curso de Ecología Humana, las habilidades de pensamiento crítico a través de la construcción de diálogos argumentativos y pragmáticos. Los foros virtuales son una estrategia didáctica que puede ser aplicada en los cursos que se implementan en entornos virtuales de aprendizaje a través de la plataforma virtual Moodle. La educación en entornos virtuales amerita que se generen trabajos cuya finalidad sea el desarrollo del pensamiento crítico $y$ el debate, debido a que es necesario atraer al estudiante a este entorno, darle las herramientas y guías necesarias para que desarrolle las actividades y alcance exitosamente las metas propuestas.

El pensamiento crítico lo define Paul y Elder (2003) como: "el modo de pensar sobre cualquier tema, contenido o problema en el cual el pensante mejora la calidad de su pensamiento al apoderarse de las estructuras inherentes del acto de pensar y al someterlas a estándares intelectuales" (pp. 4-26). En consecuencia, el acto de pensar críticamente requiere de ciertas habilidades que permiten la formulación de juicios razonables, contundentes y de carácter convincente. Además, se requiere de una reflexión de su contexto, es decir, la construcción pragmática para sugerir formas pertinentes para resolver el problema y construir argumentos que no es más que la formulación de conceptos claros que sustentan una determinada forma de pensar, con la finalidad de persuadir a otros para que acepten las ideas o los lleven a que asuman una nueva postura.

Según Van Dijk, el propósito del texto argumentativo es el de convencer. Es entonces, por eso que en la educación superior el dominio de este tipo de discurso es fundamental para enfrentar con éxito los estudios académicos. En estos escenarios mediados por las tecnologías, los estudiantes se apoyan en diversas fuentes de información, para asumir una identidad con el desarrollo de vinculaciones vivenciales de acuerdo al entorno que construye. El sujeto que lee y escribe tiene roles y una posición para interactuar y construir una comunidad letrada lo que en palabras de Flotts et. al $(2016$, p. 13) cita a Martos (2013) se define como "...Sociedad lectora, sociedad letrada o cibersociedad, todas son ámbitos posibles y necesarios de una misma praxis social indispensable para la construcción de una ciudadanía alfabetizada y crítica" (p. 10).

En busca de la construcción de una comunidad letrada en las aulas universitarias, se requiere no sólo fomentar una alfabetización funcional, es decir, prácticas tradicionales de lectura y escritura, sino que se hace indispensable una alfabetización digital que requiere de unas habilidades que permitan leer $y$ escribir en diferentes formatos y en saber seleccionar lo que se desea utilizar para contribuir a la construcción de verdaderas prácticas sociales, que contribuyan al desarrollo de habilidades de pensamiento superior. Escribir, no es una práctica que se lleva a cabo sólo desde los aspectos formales o lingüísticos del texto, esta es considerada una actividad que requiere de planificación, interpretación, inferencias, razonamiento, decodificación de la información y experiencias propias de quien lo hace. En este sentido, se requiere de estas destrezas para escribir en las redes y poder participar en los ambientes de aprendizaje que se han organizado para tal fin. De ahí que, saber escribir hoy en este tipo de entornos se constituya en un factor determinante del aprendizaje social para expandir la criticidad con la sociedad. 
Marco Tulio, R. S., Gianny, B. O., Vanessa del Carmen, P. P., Asdrubal Antonio, A. A., \& Ferley, R. G. (2020). Análisis de las habilidades de pensamiento crítico en los foros académicos virtuales. Teknos Revista Científica, 2020(2), 69-76.

Para Rolon \& Muñoz (2016), las nuevas formas de comunicación y los avances tecnológicos inciden en la formación del pensamiento del individuo, principalmente desde el desarrollo de las habilidades y capacidades dinamizadas por el epicentro escolar; de esta manera, se despliegan nuevas formas de aprender, comunicar, crear e interactuar de cara a evaluar la relación entre el pensamiento crítico y la tecnología, limitados básicamente por aspectos como la carencia institucional de recursos tecnológicos y la ausencia de políticas institucionales para su uso transversal. Como parte de una caracterización de criterios pedagógicos frente a las Tecnologías de la Información y la Comunicación (TIC), el principal reto de la educación pública es formar individuos capaces de construir conocimientos significativos y habilidades críticas que se extiendan a todas las colectividades sociales y promueven su transformación; se ejerce un nuevo rol para reconstruir, redimensionar y reformular la trascendencia del pensamiento crítico apoyado de manera sinérgica y humanizadora por la tecnología, desde el punto de vista cultural y social..

\section{Antecedentes}

\subsection{Habilidades del pensamiento crítico}

El pensamiento crítico en su estructura contiene los estándares, que se constituyen en un indicador para evaluar el pensamiento que posee una persona. En los actuales momentos, se logra evidenciar los esfuerzos que realiza la educación superior para desarrollar habilidades de pensamiento superior que conduzcan a mejorar los índices sintéticos de calidad educativa que se tienen en Colombia.

Es importante reconocer la estructura del pensamiento crítico y para observar según Paul \& Elder (2005) los estándares universales de pensamiento crítico, los cuales son: claridad, veracidad, certidumbre, precisión; relevancia, profundidad, extensión, amplitud, lógica; importancia y justicia (p.57). Pero es importante anotar que estos estándares por sí mismo no se desarrollan, es necesario que el estudiante adquiera capacidades intelectuales, referidas a los elementos que hacen posible ese pensamiento, que en la corriente de Paul y Elder estos se constituyen así: propósito del pensamiento, pregunta en cuestión, información, interpretación e inferencias, conceptos, supuestos, implicaciones y consecuencias y puntos de vista ( $p$. 56).

Basados en Halpern (2006), Saiz y Nieto (2002) y Saiz, Nieto y Orgaz (2009) se han caracterizado las habilidades de pensamiento crítico, seleccionando las dimensiones relacionadas con: razonamiento verbal, análisis de argumentos, comprobación de hipótesis, probabilidad de incertidumbre, toma de decisiones y solución de problemas. Cada una de las dimensiones tiene unos indicadores que se relacionan con las habilidades de pensamiento crítico a través de la escritura de textos.

\subsection{Foros académicos virtuales}

Con respecto a los foros virtuales, Jonassen (1998), citado por Markel (2001), indica que el foro virtual se constituye en una herramienta tecnológica que, a través de una metodología apropiada, propicia la creación de los ambientes académicos adecuados que estimulan el aprendizaje y el pensamiento crítico, a través de la negociación y construcción del conocimiento por medio del debate.

Del mismo modo, Domínguez y Alonso (2004), reafirman que el foro virtual puede llegar a desempeñar el rol central en el modelo de la enseñanza y aprendizaje orientado al desarrollo del pensamiento crítico. Además, aclara que, los foros virtuales pueden tener distintos enfoques y su valor educativo varía dependiendo de la forma de diálogo que prevalece. De acuerdo con este criterio, Arango (2004) presenta tres categorías de los foros, la primera, son foros de diálogos sociales que se caracterizan por la informalidad y la necesidad de compartir asuntos gratificantes para el autor. La segunda, los foros de diálogos argumentativos que nacen desde las lógicas individuales y se definen por la defensa de puntos de vista personales, este debate no necesariamente debe ser confrontado-con los de los demás participantes: por último, los foros de 
Marco Tulio, R. S., Gianny, B. O., Vanessa del Carmen, P. P., Asdrubal Antonio, A. A., \& Ferley, R. G. (2020). Análisis de las habilidades de pensamiento crítico en los foros académicos virtuales. Teknos Revista Científica, 2020(2), 69-76.

diálogos pragmáticos, se utilizan para construir, desde distintas miradas, significados de un mismo hecho.

Es de aclarar que, no todos los tipos de foros contribuyen de modo significativo con un constructo o con el desarrollo del pensamiento crítico de los estudiantes. Arango (2004), puntualiza que en las características del foro académico deben propiciarse la participación a través de las discusiones con argumentos y reflexiones planteadas por los participantes. Además, deben conducir hacia un diálogo pragmático donde la intención última no es persuadir al interlocutor, sino más bien indagar y utilizar el diálogo para el intercambio de pensamientos, ideas y enfoques; deben quedar expresadas las elaboraciones que conducen conocimiento, de modo que, promuevan la expresión de inquietudes que permitan identificar y proponer categorías para la discusión, valorando las diferentes propuestas, con el fin de fomentar la capacidad argumentativa de todos los participantes.

En el ámbito educativo, el ejercicio de los foros asincrónicos, propio de los foros virtuales, permite a los estudiantes articular sus ideas y opiniones desde distintas fuentes de discusión, promoviendo el aprendizaje a través de las diversas formas de interacción. (De Pepa, \& Adriana, 2006).

\section{Metodología}

Para este estudio, se utilizó una metodología cualitativa, con trabajo de campo basado en una lectura del contexto de las instituciones educativas y un análisis del contenido del texto, atendiendo al enfoque fenomenológico-hermenéutico, orientando el análisis de la descripción e interpretación de la experiencia que vivieron los estudiantes (Van Manen, 2003, pp. 108-113) en la tarea de explicar y evaluar el nivel de pensamiento crítico alcanzado por los estudiantes. Este estudio se realizó en tres momentos.

En el primero, los estudiantes conceptúan sobre los procesos de articulación de la educación ambiental en el currículo escolar y diseñan instrumentos que le han de servir para hacer una lectura de contexto, adecuada para hacer una evaluación justa.
Seguidamente, van a las instituciones educativas de la localidad y hacen una lectura de contexto basada en la observación directa y en algunas entrevistas a las personas que ellos consideraron importantes. Con estas técnicas recogieron información sobre los problemas ambientales que más afectan el contexto, las personas afectadas, los aspectos críticos y las oportunidades de mejoramiento. Para completar el diagnóstico, los estudiantes utilizaron el siguiente instrumento:

Tabla 1. Instrumento para la lectura de contextos

\begin{tabular}{|c|c|c|}
\hline $\mathbf{N}^{\circ}$ & $\begin{array}{c}\text { Indicadores } \\
\end{array}$ & Descriptivos \\
\hline 1 & $\begin{array}{l}\text { Ingreso y salida: puertas (2), } \\
\text { fachada, nombre de la institución, } \\
\text { seguridad, símbolos. }\end{array}$ & \\
\hline 2 & $\begin{array}{l}\text { Acceso a la parte administrativa y a } \\
\text { los salones: seguro, pavimentado, } \\
\text { avisos indicadores, amplios y en } \\
\text { excelente estado. Manejo excelente } \\
\text { de residuos. }\end{array}$ & \\
\hline 3 & $\begin{array}{l}\text { Planta física administrativa: } \\
\text { ubicación, pintura, señales, amplia, } \\
\text { adecuada, confortable y pertinente. } \\
\text { Manejo excelente de residuos. }\end{array}$ & \\
\hline 4 & $\begin{array}{l}\text { Aulas y laboratorios: adecuadas, } \\
\text { pintadas, con los recursos requeridos } \\
\text { y actualizados. Manejo excelente de } \\
\text { residuos. }\end{array}$ & \\
\hline 5 & $\begin{array}{l}\text { Predios: Amplios, arborizados, con } \\
\text { señales, con parques recreacionales } \\
\text { y didácticos. Manejo excelente de } \\
\text { residuos. }\end{array}$ & \\
\hline 6 & $\begin{array}{l}\text { Instalaciones deportivas: adecuadas } \\
\text { y suficientes. Manejo excelente de } \\
\text { residuos. }\end{array}$ & \\
\hline 7 & $\begin{array}{l}\text { Tienda escolar: manejo de residuos, } \\
\text { señales y orientación pedagógica. }\end{array}$ & \\
\hline 8 & $\begin{array}{l}\text { Restaurante escolar: ambiente, } \\
\text { manejo de residuos, manejo de } \\
\text { plagas, preparación de los alimentos } \\
\text { y atención a los niños. }\end{array}$ & \\
\hline 9 & $\begin{array}{l}\text { Recursos para el manejo de } \\
\text { residuos: canecas, carretillas, bolsas } \\
\text { plásticas, etc. }\end{array}$ & \\
\hline 10 & $\begin{array}{l}\text { Proyecto ambiental escolar PRAE: } \\
\text { aplicación, pertinencia y apropiación. }\end{array}$ & \\
\hline
\end{tabular}

Descripción del estado en que se encuentran cada uno de los indicadores en la lectura de contexto que se hizo

Por último, con la información compilada, elaboraron un texto argumentativo que fue presentado, criticado y evaluado en los foros virtuales que estaban destinados para tal fin. Este texto 
Marco Tulio, R. S., Gianny, B. O., Vanessa del Carmen, P. P., Asdrubal Antonio, A. A., \& Ferley, R. G. (2020). Análisis de las habilidades de pensamiento crítico en los foros académicos virtuales. Teknos Revista Científica, 2020(2), 69-76.

producido por los estudiantes y el docente, fue el insumo de este trabajo investigativo. Con el propósito de determinar el grado de complejidad discursiva de los informantes, se siguió una técnica cualitativa, aplicando los procedimientos de la grounded theory (Glaser y Strauss, 1967), a través del software Atlas/ti, haciendo un análisis del contenido del texto. Para ello, se utilizaron las categorías preestablecidas en el diseño del instrumento que permitió hacer la lectura de contexto y la información compilada a través de algunas entrevistas. De ellas se derivaron otras, que emergieron del contenido del texto. De esta información, los estudiantes informantes hicieron un informe con predominancia de las secuenciaciones argumentativas (Adam, 1992), que sirvieron para evaluar el desarrollo del pensamiento crítico.

\section{Análisis y discusión de resultados}

Para el análisis del texto se utilizaron dos grandes categorías: Prácticas ambientalistas y articulación de la dimensión ambiental en el currículo escolar. Cada una de ellas, por definición tiene unas subcategorías que fueron tenidas en cuenta en el diseño de los protocolos de investigación. La primera de ellas: articulación de la dimensión ambiental al currículo, es definida desde los atributos de la misma, considerando su existencia desde la coherencia con la política educativa, su pertinencia con el contexto, su trascendencia en la resolución de los problemas ambientales y en la promoción de una cultura ambiental que se ponga en evidencia en cada una de las gestiones escolares (Torres, M., 2002) Las prácticas ambientales son un conjunto de acciones sencillas que implican un cambio de actitud y de comportamiento en nuestras actividades diarias promoviendo una relación amigable con el ambiente. Con la aplicación de buenas prácticas podemos alcanzar rápidos y sorprendentes resultados con pequeños cambios, el aprovechamiento de los recursos como el agua, la energía eléctrica, el transporte, la gestión adecuada de residuos y políticas de compras sustentables. Estas prácticas generan beneficios ambientales, sociales económicos y reducen nuestra huella ecológica (Barrera, M., 2011). De la revisión del texto, se encontró que:

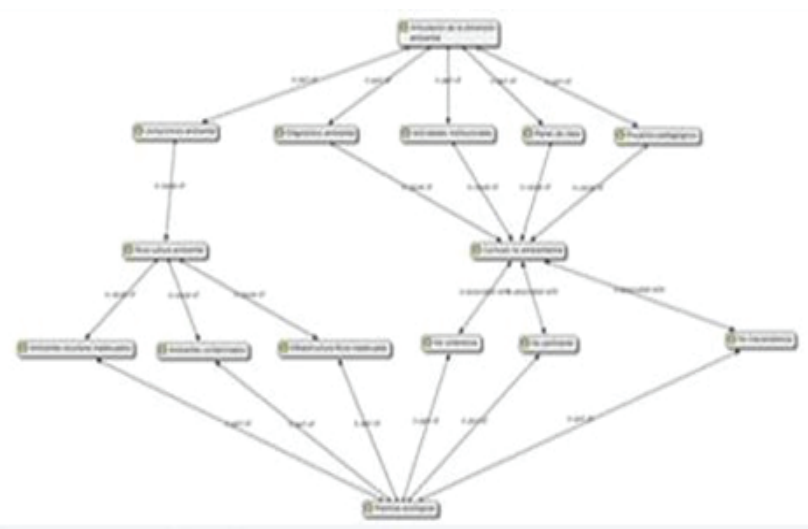

Figura 1. Esquema argumentativo (Adam, 1992): Prácticas Ambientales

De la estructura semántica anterior, se hizo el siguiente Memorándum: La articulación de la educación ambiental al currículo escolar, depende de la coherencia con las políticas educativas y con la responsabilidad institucional explicitada en la misión, la pertinencia de sus prácticas ambientales y de la trascendencia de las mismas en el contexto en que se encuentran las instituciones educativas, contribuyendo de manera significativa en la resolución de los problemas.

Sin embargo, en las instituciones educativas visitadas, se encontró que se implementan algunas actividades que buscan promover la educación ambiental, pero que no son pertinentes porque no obedecen a un diagnóstico que se haya realizado para la identificación y priorización de los problemas ambientales del contexto. Tampoco son coherentes con la política ambiental porque no se evidencia la formación de una cultura en las personas que comprenda y actúe en defensa de las condiciones de vida del planeta, promovida desde la transversalidad curricular del proyecto ambiental escolar. $Y$ al revisar la trascendencia de las prácticas ambientales que se implementan en las instituciones educativas, por su carácter de ser eventuales, no planeadas y de poco impacto, porque no salen del interior de las instituciones educativas. 
Marco Tulio, R. S., Gianny, B. O., Vanessa del Carmen, P. P., Asdrubal Antonio, A. A., \& Ferley, R. G. (2020). Análisis de las habilidades de pensamiento crítico en los foros académicos virtuales. Teknos Revista Científica, 2020(2), 69-76.

Por las razones expuestas anteriormente, la dimensión ambiental no está articulada en el currículo escolar y en consecuencia las prácticas ambientales que se implementan no son el medio para formar a una persona con una cultura ambiental que evidencie en cada una de sus acciones, el nivel de conciencia alcanzado.

Esa estructura argumentativa fue evaluada teniendo en cuenta las habilidades de pensamiento listadas por Halpern (2006), Saiz y Nieto (2002) y Saiz, Nieto y Orgaz (2009), puesta en una matriz, con las adaptaciones que hizo el grupo investigador para evaluar el nivel de pensamiento crítico alcanzado por los estudiantes en su lectura de contexto, producción textual y en el debate presentado en el foro virtual que fue habilitado para tal fin.

Tabla 2. Matriz para evaluar el pensamiento crítico de los estudiantes

\begin{tabular}{|c|c|c|}
\hline $\begin{array}{c}\text { Dimensión de } \\
\text { pensamiento } \\
\text { crítico }\end{array}$ & Microrrelato & Indicador \\
\hline $\begin{array}{l}\text { Formulación } \\
\text { de hipótesis }\end{array}$ & $\begin{array}{l}\text { Esta etapa hizo parte de los } \\
\text { procesos de articulación de la } \\
\text { dimensión ambiental en el } \\
\text { currículo escolar a la luz del } \\
\text { texto de Torres M. (2013) y el } \\
\text { [14] análisis de los PRAE de } \\
\text { Sincelejo. La tarea consistió } \\
\text { en formular una hipótesis y } \\
\text { diseñar un instrumento que } \\
\text { orientará la lectura de } \\
\text { contexto indagado. }\end{array}$ & $\begin{array}{c}\text { Formula una } \\
\text { hipótesis } \\
\text { sobre el } \\
\text { estado en que } \\
\text { se encuentran } \\
\text { los PRAE en } \\
\text { Sincelejo. }\end{array}$ \\
\hline $\begin{array}{l}\text { Descripción } \\
\text { del contexto }\end{array}$ & $\begin{array}{c}\text { p.14 2:5 "que tanto en la } \\
\text { misión como en la visión no } \\
\text { aparece la dimensión } \\
\text { ambiental" } \\
\text { p.14 2:13 "es la poca cultura } \\
\text { ecológica la que provoca un } \\
\text { encadenamiento de } \\
\text { causas y efectos." } \\
\text { p.14 2:28 (...también } \\
\text { podemos decir que hay aulas } \\
\text { que están en mal estado, no } \\
\text { están condicionadas para el } \\
\text { trabajo } \\
\text { académico...) } \\
\text { p.14.2:16 (...mucha } \\
\text { acumulación de residuos sin } \\
\text { tener en cuenta los } \\
\text { recipientes habilitados para } \\
\text { tal fin...) } \\
\text { p.14.2:17 “...la poca cultura } \\
\text { ecológica que se evidencia en } \\
\text { el contexto..." }\end{array}$ & $\begin{array}{l}\text { Describe las } \\
\text { condiciones } \\
\text { en que se } \\
\text { encuentran } \\
\text { los aspectos } \\
\text { indagados en } \\
\text { el contexto. }\end{array}$ \\
\hline
\end{tabular}

\begin{tabular}{|c|c|c|}
\hline & $\begin{array}{c}\text { p.16 2:18 “Los docentes } \\
\text { predican, pero no aplican” } \\
\text { p.2 2:32 (“... los ambientes } \\
\text { escolares estaban } \\
\text { inadecuados para el trabajo } \\
\text { óptimo de los procesos de } \\
\text { enseñanza y de } \\
\text { aprendizaje....) }\end{array}$ & \\
\hline $\begin{array}{c}\text { Análisis de } \\
\text { argumentos }\end{array}$ & $\begin{array}{c}\text { Del análisis del contenido del } \\
\text { texto, se encuentra que: } \\
\text { Análisis de pertinencia: } \\
\text { p.26 2:6:(...las actividades } \\
\text { ambientales no obedecen a un } \\
\text { diagnóstico...) } \\
\text { Análisis de Coherencia } \\
\text { p.2 } 2: 5 \text { (...que tanto en la } \\
\text { misión como en la visión no } \\
\text { aparecen aspectos } \\
\text { relacionados con lo } \\
\text { ambiental...) } \\
\text { Análisis de Trascendencia } \\
\text { p.12 } 2: 13 \text { (...la falta de } \\
\text { conciencia ecológica y de una } \\
\text { cultura } \\
\text { ambiental...) } \\
\text { p.12 2:40 (...por esto no se } \\
\text { abordan los problemas } \\
\text { ambientales del } \\
\text { contexto...) } \\
\text { De lo anterior, se extrae } \\
\text { que: Prácticas ecológicas no } \\
\text { pertinentes, no coherentes, no } \\
\text { trascendentes que } \\
\text { conllevaron a establecer una } \\
\text { cultura ambiental no } \\
\text { adecuada en ese contexto y } \\
\text { una conciencia ecológica no } \\
\text { proactiva. }\end{array}$ & $\begin{array}{c}\text { Establece } \\
\text { relaciones causa- } \\
\text { efecto y } \\
\text { da explicaciones } \\
\text { sobre el hecho } \\
\text { observado. } \\
\end{array}$ \\
\hline $\begin{array}{l}\text { Comproba- } \\
\text { ción de } \\
\text { hipótesis }\end{array}$ & $\begin{array}{c}\text { Las instituciones educativas } \\
\text { estudiadas no tienen articulada } \\
\text { la dimensión ambiental en su } \\
\text { currículo porque } \\
\text { Sus prácticas ambientales no } \\
\text { son pertinentes } \\
\text { Sus prácticas ambientales no } \\
\text { son coherentes con la misión } \\
\text { de la escuela y las políticas } \\
\text { educativo-ambientales Sus } \\
\text { prácticas ambientales no son } \\
\text { trascendentes en el contexto } \\
\text { que se está estudiando }\end{array}$ & $\begin{array}{l}\text { Presenta } \\
\text { argumentos } \\
\text { lógicos y } \\
\text { consistentes que } \\
\text { ponen en } \\
\text { evidencia la } \\
\text { hipótesis }\end{array}$ \\
\hline $\begin{array}{l}\text { Probabilidad } \\
\text { de } \\
\text { incertidum- } \\
\text { bres }\end{array}$ & $\begin{array}{c}\text { Si tuvieran articulada la } \\
\text { dimensión ambiental en el } \\
\text { currículo, sus planes de aula, } \\
\text { proyectos pedagógicos, } \\
\text { actividades institucionales y } \\
\text { compromisos misionales } \\
\text { estuvieran en función de } \\
\text { resolver los problemas } \\
\text { ambientales más críticos en el } \\
\text { entorno. }\end{array}$ & \begin{tabular}{|c|} 
Plantea una \\
postura diferente \\
a la tesis y \\
cuestiona sus \\
inconsistencias
\end{tabular} \\
\hline
\end{tabular}


Marco Tulio, R. S., Gianny, B. O., Vanessa del Carmen, P. P., Asdrubal Antonio, A. A., \& Ferley, R. G. (2020). Análisis de las habilidades de pensamiento crítico en los foros académicos virtuales. Teknos Revista Científica, 2020(2), 69-76.

\begin{tabular}{|c|c|c|}
\hline $\begin{array}{c}\text { Toma de } \\
\text { decisiones }\end{array}$ & $\begin{array}{c}\text { Las prácticas ecológicas son } \\
\text { no pertinentes, no coherentes } \\
\text { con la política y misión de la } \\
\text { escuela ni son trascendentes } \\
\text { en el impacto que producen. }\end{array}$ & $\begin{array}{c}\text { Emite un juicio de } \\
\text { valor sobre el } \\
\text { estado en que se } \\
\text { encuentra el } \\
\text { contexto y su } \\
\text { relación con las } \\
\text { prácticas } \\
\text { ecológicas }\end{array}$ \\
\hline $\begin{array}{c}\text { Solución del } \\
\text { problema }\end{array}$ & $\begin{array}{c}\text { Las prácticas ecológicas no } \\
\text { están en función de la } \\
\text { resolución del problema del } \\
\text { agua en Sincelejo. Se deben } \\
\text { formular, definir y promover } \\
\text { atendiendo a un diagnóstico } \\
\text { ambiental. }\end{array}$ & $\begin{array}{c}\text { Sugiere acciones } \\
\text { pertinentes para } \\
\text { resolver el } \\
\text { problema }\end{array}$ \\
\hline
\end{tabular}

Fuente: Elaboración propia. Apoyado en Halpern (2006), Saiz y Nieto (2002) y Saiz, Nieto y Orgaz (2009).

De acuerdo con la red semántica (figura 1), el memorándum y el análisis del contenido del texto que se presenta en la tabla 1, sirven para evidenciar el nivel de pensamiento crítico, alcanzado durante un proceso que cuenta con fases clave, como la planeación y aprendizaje de la estrategia, indagación en el contexto escolar y la última que busca la producción de un texto argumentativo que ponga en evidencia la confirmación o la falsedad de la hipótesis planteada. En el texto, es clara la elaboración de argumentos en contra de la hipótesis planteada, encontrando que la evaluación de la coherencia, pertinencia y trascendencia de las prácticas ambientales de algunas instituciones educativas, de acuerdo con Torres M., (2002), es notoria la ausencia de una conceptualización clara, con respecto al ambiente y a la educación ambiental, lo que ha llevado a la formulación de objetivos demasiado generales y al desarrollo de estrategias imprecisas (dirigidas a la realización de acciones puntuales y aisladas), desde las propuestas o proyectos, que se implementan, lo cual no permite el logro de los impactos requeridos, en materia de formación para un manejo adecuado del ambiente.

Además, hay una clara descontextualización de las acciones realizadas en materia de educación ambiental, debido a que en la mayoría de los casos dichas acciones no parten de los diagnósticos o perfiles ambientales regionales, o locales, ni se relacionan con los planes regionales $u$ otros instrumentos de planeación (POT, Planes de
Desarrollo, Planes Trianuales de las Corporaciones Autónomas y Planes de Gestión Ambiental Regional, entre otros). Aquí también se detecta la ausencia de proyección de la visión sistémica del ambiente, a los análisis de los problemas ambientales. Finalmente, Esto trae como consecuencia la aplicación de concepciones y de proyecciones diversas (contradictorias y en ocasiones opuestas), en las acciones que los actores educativos desarrollan en la temática particular, contribuyendo así a la atomización y los bajos impactos en la construcción de una cultura ambiental en el seno de las instituciones educativas.

Siguiendo la línea argumentativa presentada en el texto, se observa una secuencia, que en primera instancia, describe el estado en que se encuentra el contexto en materia ambiental, mostrando fotos que ponen en evidencia la descripción, seguidamente presentan agentes causales y algunas consecuencias que se derivan del hecho, y finalmente hacen una evaluación del estado en que se encuentran las instituciones justificando la emisión del juicio realizado, haciendo recomendaciones ajustadas a lo encontrado y a la política ambiental vigente. Este tipo de texto fue sometido a la crítica de los participantes en el foro, dándole oportunidad para que los aportantes pudieran aclarar, defender y argumentar sus posturas y decisiones. Este tipo de lógica racional, se ajusta a una forma de pensar y de construir argumentos a favor o en contra de los juicios de valor que se hacen al analizar, entender y evaluar el contenido de un texto, dejando al descubierto la forma de utilizar un medio como lo es un foro virtual para promover el desarrollo del pensamiento crítico.

\section{Conclusiones}

Evaluar el pensamiento crítico a través de la escritura digital es una tarea compleja, debido a que el ejercicio implica las relaciones más profundas entre el pensamiento y lenguaje. Se analizan las habilidades de pensamiento crítico a través de la estrategia de los foros académicos en el curso de Ecología Humana, logrando las siguientes consideraciones: los estudiantes en los diferentes análisis de los microrrelatos realizan afirmaciones que están 
Marco Tulio, R. S., Gianny, B. O., Vanessa del Carmen, P. P., Asdrubal Antonio, A. A., \& Ferley, R. G. (2020). Análisis de las habilidades de pensamiento crítico en los foros académicos virtuales. Teknos Revista Científica, 2020(2), 69-76.

respaldadas por unas evidencias que se muestran a través de indicadores, lo cual permite generar conclusiones en su mayoría justificadas para la toma de decisiones y la solución de problemas, evidenciando las habilidades de pensamiento crítico en el texto que se genera a través del diálogo argumentativo entre los participantes.

El escenario foro virtual, se constituye en un medio en el que se construye un texto argumentativo a través del debate entre los participantes. De la crítica a la que es sometida cada proposición y de la consistencia y contundencia de cada argumento para defender cada concepto y posición, depende la secuencia lógica del argumento y desarrollo del pensamiento del más alto nivel.

\section{Referencias}

Adam. Les textes: types et prototypes. Paris: Nathan. 1992 Álvarez, G. Y. C. (2013). La evaluación de las habilidades del pensamiento crítico asociadas a la escritura digital. Revista Virtual Universidad Católica del Norte, 3(40), 68-83.

Arango, A M. (2004, abril). Foros virtuales como estrategia de aprendizaje. Revista Debates Latinoamericanos, $\mathrm{N}^{\circ}$ 2. 2004. Recuperado el 28 de marzo de 2006 De http://www.rlcu.org.ar/revista/numeros/02-02-Abril2004/documentos/Arango.pdf. 2004.

Barrera Vélez, M. (2011). Programa de educación ambiental no formal en el establecimiento penitenciario de mediana seguridad y carcelario de Cali-EPMSCCAL (Bachelor's thesis, Universidad Autónoma de Occidente). en: http://bdigital.uao.edu.co/bitstream/10614/1636/1/TAA 00770.pdf

De Pepa, M., \& Adriana, M. (2006). La utilización de foros virtuales en la universidad como metodología de aprendizaje colaborativo. Revista cognición, 8, 59-74.

Flotts, M. P., Manzi, J., Lobato, P., Durán, M. I., Díaz, M. P., \& Abarzúa, A. (2016). Aportes para la enseñanza de la escritura.: tercer estudio regional comparativo y explicativo. OREALC/UNESCO

Glaser, B., \& Strauss, A. (1967). El desarrollo de la teoría fundada.Chicago, EEUU: Aldine.

Halpern, D. (2006). Halpern critical thinking assessment using everyday situations: background and scoring standards $\left(2^{\circ}\right.$ Report). Unpublished manuscript. Claremont, CA: Claremont McKenna College.
Paul, R., \& Elder, L. (2003). La mini-guía para el pensamiento crítico, conceptos y herramientas. California: Fundación para el pensamiento crítico.

Rolon, M., \& Muñoz, J. G. (2016, October). El pensamiento crítico frente a las tecnologías de información y comunicación en la educación media. In 3er Simposio Internacional y 4to Coloquio Regional de Investigación Educativa y Pedagógica.

S, Markel, S. L., \& ECl, E. E. (2001). Technology and education online discussion forums. Online journal of distance learning administration, 4., núm., II, summer 2001, Recuperado 30/03/2005 http://www.westga.edu/ distance/ojdla/summer42/ma rkel42.htmlDomínguez

Torres Carrasco, M. (2002). Reflexión y Acción: El diálogo fundamental para la Educación Ambiental. Serie Documentos Especiales, Pedagogía y Ambiente, 2.

Van Dijk, T. A. (2011). Discurso y poder. Editorial Gedisa.

Van Manen, M. (2003). Investigación educativa y experiencia vivida: ciencia humana para una pedagogía de la acción y la sensibilidad. Idea books. 